\title{
Inflation in the closed FLRW model and the $\mathrm{CMB}$
}

\author{
Béatrice Bonga, ${ }^{1}$ Brajesh Gupt, ${ }^{2}$ Nelson Yokomizo $^{3}$ \\ Institute for Gravitation and the Cosmos \& Physics Department, The Pennsylvania State \\ University, University Park, PA 16802 U.S.A. \\ E-mail: bpb165@psu.edu,bgupt@gravity.psu.edu, yokomizo@gravity.psu.edu
}

\begin{abstract}
Recent cosmic microwave background (CMB) observations put strong constraints on the spatial curvature via estimation of the parameter $\Omega_{\mathrm{k}}$ assuming an almost scale invariant primordial power spectrum. We study the evolution of the background geometry and gauge-invariant scalar perturbations in an inflationary closed FLRW model and calculate the primordial power spectrum. We find that the inflationary dynamics is modified due to the presence of spatial curvature, leading to corrections to the nearly scale invariant power spectrum at the end of inflation. When evolved to the surface of last scattering, the resulting temperature anisotropy spectrum $\left(C_{\ell}^{\mathrm{TT}}\right)$ shows deficit of power at low multipoles $(\ell<20)$. By comparing our results with the recent Planck data we discuss the role of spatial curvature in accounting for CMB anomalies and in the estimation of the parameter $\Omega_{\mathrm{k}}$. Since the curvature effects are limited to low multipoles, the Planck estimation of cosmological parameters remains robust under inclusion of positive spatial curvature.
\end{abstract}




\section{Contents}

1 Introduction 1

2 Preliminaries 3

2.1 Background dynamics 4

2.2 Perturbations 5

3 Initial conditions $\quad 8$

4 Results 11

4.1 Why does spatial curvature matter? 11

4.2 Power spectrum 12

$\begin{array}{lll}4.3 & \text { Temperature anisotropy spectrum } & 15\end{array}$

5 Discussion $\quad 17$

6 Acknowledgements $\quad 18$

A Hyperspherical harmonics and linear perturbations on $\mathbb{S}^{3} \quad 18$

\section{Introduction}

The recent measurements of the cosmic microwave background radiation (CMB) anisotropies by observational missions such as Planck [1] and WMAP [2] are in excellent agreement with the well known $\Lambda$ CDM model of the Universe. According to this model, the geometry of spacetime is described by a homogeneous and isotropic Friedmann-Lemaittre-Robertson-Walker (FLRW) metric, and the energy content of the Universe is composed of baryonic and cold dark matter $\left(\Omega_{\mathrm{m}}\right)$, radiation $\left(\Omega_{\mathrm{r}}\right)$, a cosmological constant $\left(\Omega_{\Lambda}\right)$ and spatial curvature $\left(\Omega_{\mathrm{k}}\right)$ [1-5]. Within this scenario, the observational data imposes strong constraints on the spatial curvature of the FLRW background: $\Omega_{\mathrm{k}}=-0.005_{-0.017}^{+0.016}$ from Planck measurements alone, and $\Omega_{\mathrm{k}}=0.000_{-0.005}^{+0.005}$ when the Planck measurements are combined with BAO data [6] (results are quoted at 95\% confidence intervals). The origin of the tiny temperature fluctuations in the $\mathrm{CMB}$ can be attributed to almost scale invariant primordial density perturbations predicted by inflation. In the simplest inflationary scenario the accelerated expansion of the Universe is driven by a single scalar field $\phi$ with standard kinetic energy slowly rolling down a potential $V(\phi)$ in a spatially flat $\left(\Omega_{\mathrm{k}}=0\right)$ FLRW spacetime [3, 4, 7-11].

While consistent with a spatially flat spacetime geometry, the current bounds on $\Omega_{\mathrm{k}}$ allow for the possibility that we live in a slightly curved patch of the Universe, which can be related to a global non-zero spatial curvature, e.g. open or closed FLRW spacetime, or to the effect of super-horizon fluctuations on our local patch in a globally flat Universe [6]. Closed FLRW model has also been argued to be of phenomenological [12] and fundamental importance [13]. In the presence of spatial curvature, the predictions of inflationary models are affected in two distinct ways. First, the transfer functions encoding the evolution of linear perturbations from the end of inflation until today are modified by the presence of curvature terms in the Boltzmann equations. This effect is well understood and is fully implemented in the Boltzmann codes employed for the 
integration of these equations (as CAMB ${ }^{1}$, for instance [14]). Second, the spectrum of primordial perturbations can be affected by the presence of curvature during inflation. Recall that the rapid accelerated expansion of the Universe during inflation 'washes away' any spatial curvature that might have been present before inflation, making the spatial geometry at the end of inflation very close to flat. Therefore, even if the spatial curvature $\Omega_{\mathrm{k}}$ is very small at the end of inflation and today, it might have been significant during the early stages of inflation. In this scenario, the evolution of quantum perturbations in a spatially curved spacetime is distinct from that in a spatially flat spacetime during inflation, especially near the onset of inflation. As a result, the spectrum of primordial fluctuations will be influenced by the effects of $\Omega_{\mathrm{k}} \neq 0$. Significant deviations from the simple power law form generally can occur for wavelengths comparable to the curvature radius, which are later imprinted as scale-dependent features in the CMB power spectra. This effect, however, has not been analyzed in great detail, even for small spatial curvatures, $\Omega_{\mathrm{k}}$. In particular, these potential features in the primordial power spectrum are not taken into account for the estimation of cosmological parameters including $\Omega_{\mathrm{k}}$ in Planck analysis $[6,15]$, which is based on a tilted power law spectrum for the primordial scalar perturbations. The goal of this paper is to revisit these issues for the closed FLRW model, i.e. with $\Omega_{\mathrm{k}}<0$, by studying the evolution of quantum perturbations in a closed inflationary Universe.

In a closed FLRW model the topology of the spacetime is $\mathbb{R} \times \Sigma$ with the topology $\Sigma$ of the spatial sections that of a 3 -sphere $\Sigma=\mathbb{S}^{3}$. In this case, the angular scales in the CMB that are potentially affected by the global curvature can be estimated by setting $\Omega_{\mathrm{k}}$ to the observed values. Using the Planck estimate for the Hubble constant $H_{0}=(67.8 \pm 0.9) \mathrm{km} \mathrm{s}^{-1}$ $\mathrm{Mpc}^{-1}$ and $\Omega_{\mathrm{k}}=-0.005$ [6], we find that the radius of the spatial $\mathbb{S}^{3}$ section of the Universe today is: $r_{0} \simeq 4.5 r_{L S}$, where $r_{L S} \approx 14 \mathrm{Gpc}$ is the radius of the last scattering surface. ${ }^{2}$ This indicates that curvature effects, if present, will be more pronounced at length scales comparable to that of the CMB sky. These scales correspond to the lowest multipoles. Interestingly, recent CMB observations reveal anomalies in the temperature anisotropy spectrum at low multipoles including a power suppression for $\ell \lesssim 30$ that is mildly incompatible with the predictions of an almost scale-invariant primordial spectrum $[1,16,17]$. Therefore, it is natural to ask to what extent spatial curvature can affect the low- $\ell$ spectrum and account for this anomaly.

The power suppression at low multipoles has been studied in the case of open inflation, with the help of toy models, by introducing an early period of fast-roll at the onset of inflation following the process of bubble nucleation $[18,19]$. A similar mechanism involving a transient regime of fast-rolling has also been advocated in flat models [20-25], showing that the presence of curvature may not be essential in this picture. In both cases, however, one needs to include finetuned localized features in the potential energy $V(\phi)$ of the scalar field on scales corresponding to the present day Hubble horizon. In this paper, we focus on the less studied case of a closed Universe for the quadratic and Starobinsky potentials without extra features.

Due to its finite size, a closed Universe has a characteristic length scale given by the curvature radius $r_{0}$. It is generally expected that the primordial fluctuations truncate on such a scale, which thus acts as a natural infrared cutoff [26-31]. Under this assumption, it was shown in [29] that the quadrupole anomaly can be consistently interpreted as a curvature effect. Evidence for such a behavior was presented in [32], where it was found that the power spectrum of scalar perturbations on a closed de Sitter background is slightly suppressed at the scale of the curvature radius. This raises the question as to whether the full inflationary dynamics can

\footnotetext{
${ }^{1}$ http://camb.info

${ }^{2}$ Note that $r_{0}$ is the radius of a 3-sphere representing the whole Universe and $r_{L S}$ is radius of the 2-sphere corresponding to the surface of last scattering.
} 
actually enforce the conjectured truncation and how exactly the primordial power spectrum departs from the power law characteristic of flat spaces at the length scale set by $r_{0}$.

In order to investigate these questions, we perform a detailed analysis of the inflationary evolution of gauge-invariant scalar perturbations in a closed FLRW Universe for a scalar field with a quadratic and Starobinsky potential, based on the Hamiltonian formalism of [33]. We first determine initial conditions for the background geometry which lead to a sufficiently long slow-roll phase that is compatible with observations. Note that it is a priori not clear if a given initial condition will lead to inflation at all [34]. Providing initial conditions for the perturbations at the onset of inflation, we numerically integrate these equations of motion for a range of values of $\Omega_{\mathrm{k}}$ including the Planck estimates, $\Omega_{\mathrm{k}}=-0.005$. Since the curvature term in the Friedmann equation scales as $a^{-2}$, where $a$ is the scalar factor, the background evolution is strongly affected by the spatial curvature in the early stages of inflation, leading to a breakdown of the slow-roll approximation [26]. This mechanism allows for a transient fast-roll regime without the need of modifying the potential, with significant implications for the modes crossing the horizon at these times. We calculate the power spectrum at the end of inflation and use the Boltzmann code CAMB to determine the spectrum of temperature anisotropies in the CMB. We find that the modifications in the primordial power spectrum lead to power suppression at multipoles $\ell \lesssim 20$, with almost $30 \%$ suppression at the lowest multipole, $\ell=2$. This indicates that, while this suppression of power is not strong enough to completely explain the observed anomaly, the presence of spatial curvature can partially account for it. Also, we find that the modification in the power spectrum has negligible effects on the estimation of cosmological parameters. This happens because the curvature effects on the CMB are limited to only low $\ell$ 's while for $\ell>20$ the spectrum remains practically unaffected. Therefore, while a priori being conceptually inconsistent, the Planck estimation of cosmological parameters in the presence of spatial curvature [6] is phenomenologically robust.

The paper is organized as follows. In section 2 we provide a brief overview of the equations of motion of the closed FLRW model in the presence of a scalar field with a potential $V(\phi)$ and gauge-invariant scalar perturbations on it. Section 3 provides details on the initial conditions for the background variables and quantum perturbations chosen such that the future evolution has a minimal duration of inflation and leads to a power spectrum compatible with observations at large $\ell$ 's. We study the evolution of the background spacetime and scalar perturbations in section 4. This is where we compute the temperature anisotropy spectrum and discuss the effects of spatial curvature on the CMB spectrum and estimation of cosmological parameters. We conclude in section 5 with a summary of the main results and future outlook. Technical details about the properties of the spherical harmonics are given in the appendix A.

\section{Preliminaries}

This section is divided into two subsections. In the first subsection we provide a brief overview of the background spacetime dynamics of a closed FLRW spacetime in the presence of a nonminimally coupled scalar field with a self-interacting potential. In the second subsection, we discuss the construction of gauge-invariant linear scalar perturbations $q$ using Hamiltonian methods described in [33], obtain the second order Hamiltonian [33, 35, 36] and derive the equations of motion for $q$. 


\subsection{Background dynamics}

For the background, we consider a homogeneous, isotropic spatially curved Friedmann-LemaitreRobertson-Walker model with topology $\mathbb{R} \times \mathbb{S}^{3}$. The spacetime metric is

$$
d s^{2}=-d t^{2}+\tilde{a}^{2}(t) r_{o}^{2} d \Omega^{2}
$$

where $r_{o}$ is the curvature radius of the $\mathbb{S}^{3}$ spatial section, $\Omega_{i j}$ is the metric on the fiducial unit three-sphere with volume $\mathcal{V}_{o}=2 \pi^{2}$ and $\tilde{a}$ is dimensionless scale factor. In spherical coordinates $(\chi, \theta, \varphi)$, the fiducial three-sphere metric representing the spatial section takes the form:

$$
d \Omega^{2}=d \chi^{2}+\sin ^{2} \chi\left(d \theta^{2}+\sin ^{2} \theta d \varphi^{2}\right) .
$$

It is convenient to define the dimensionful scale factor: $a(t):=r_{o} \tilde{a}(t)$. Given a matter field with the energy density $\rho$ and pressure $P$, the dynamics of $a(t)$ is given by the Friedmann and Raychaudhuri equations:

$$
\begin{aligned}
H^{2} & =\frac{8 \pi G}{3} \rho-\frac{1}{a^{2}}, \\
\dot{H} & =-4 \pi G(\rho+P)+\frac{1}{a^{2}},
\end{aligned}
$$

where $H=\dot{a} / a$ is the Hubble parameter. We are interested in the inflationary dynamics which is driven by a scalar field with a self-interacting potential $V(\phi)$. The energy density $\rho$ and the pressure $P$ of the scalar field are:

$$
\rho=\frac{1}{2} \dot{\phi}^{2}+V(\phi) \quad \text { and } \quad P=\frac{1}{2} \dot{\phi}^{2}-V(\phi) .
$$

Using the above definitions and equations (2.3) and (2.4) we obtain the Klein-Gordon equation which governs the evolution of the scalar field:

$$
\ddot{\phi}+3 H \dot{\phi}+\frac{d V}{d \phi}=0
$$

Among the eqs. (2.3), (2.4) and (2.6) only two are independent: any of these equations can be derived by combining the other two. Therefore, it is sufficient to consider the Klein-Gordon equation (2.6) together with the Friedmann equation (2.3) to completely describe the evolution of the background spacetime. These two equations form a well-posed initial value problem, which - given proper initial conditions at an initial time - can be solved to evaluate the quantities $a(t)$ and $\phi(t)$ at a later (or earlier) time. In this paper, we consider two different potentials for the inflationary phase: the quadratic potential

$$
V(\phi)=\frac{1}{2} m^{2} \phi^{2}
$$

and the Starobinsky potential [37-40]

$$
V(\phi)=\frac{3 M^{2}}{32 \pi G}\left(1-e^{-\sqrt{\frac{16 \pi G}{3}} \phi}\right)^{2} .
$$

In both cases the values of the mass parameters $m$ and $M$ are fixed by using Einstein's equations in conjugation with recent observations as described in section 3. 
After inflation ends, the scalar field decays completely and standard model particles are created during the reheating phase. The further evolution of the background spacetime is described by the standard $\Lambda$ CDM model: the matter content of the Universe consists of baryonic and cold dark matter $(w:=P / \rho=0)$, radiation $(w=1 / 3)$ and the cosmological constant or dark energy $(w=-1)$. The Friedmann equation describing the background geometry in this phase all the way till today is:

$$
H^{2}=H_{0}^{2}\left(\Omega_{\mathrm{m}}\left(\frac{a}{a_{0}}\right)^{-3}+\Omega_{\mathrm{r}}\left(\frac{a}{a_{0}}\right)^{-4}+\Omega_{\Lambda}+\Omega_{\mathrm{k}}\left(\frac{a}{a_{0}}\right)^{-2}\right),
$$

where $H_{0}$ is the Hubble parameter today. The parameters $\Omega_{\mathrm{m}}=0.31, \Omega_{\mathrm{r}}=9.2 \times 10^{-5}, \Omega_{\Lambda}=$ 0.69 and $\Omega_{\mathrm{k}}$ are the contribution to the total extrinsic curvature from matter, radiation, dark energy and spatial curvature, respectively, whose values are measured today by the recent CMB experiments. For the closed FLRW model under consideration, $\Omega_{\mathrm{k}}<0$. The Planck power spectra constrain $\Omega_{\mathrm{k}}=-0.005_{-0.017}^{+0.016}$ at a $95 \%$ confidence interval. Adding data from BAO to break the 'geometric degeneracy' between $H_{0}$ and $\left|\Omega_{\mathrm{k}}\right|$, yields $\Omega_{\mathrm{k}}=0.000_{-0.005}^{+0.005}$ at the same confidence interval [6].

\subsection{Perturbations}

On the homogeneous and isotropic inflationary background described above, we introduce first order, purely inhomogeneous perturbations: ${ }^{3}$

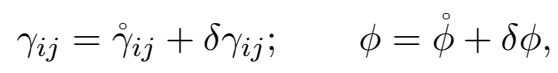

where ${ }_{i j}=a^{2} \Omega_{i j}$ is the background metric, $\dot{\phi}$ is the background homogeneous inflaton field, and $\delta \gamma_{i j}$ and $\delta \phi$ are the inhomogeneous metric and inflaton perturbations respectively. The metric perturbation $\delta \gamma_{i j}$ has two degrees of freedom, let's denote them $\gamma^{(1)}$ and $\gamma^{(2)}$ (see appendix A for exact relation), which are equivalent to the two Bardeen potentials (usually denoted by $\Psi$ and $\Phi$ in the literature $[41,42]$ ). Paying attention to the symmetries of the spatial hypersurface we expand these perturbations in scalar harmonic functions $Q_{n l m}$ and tensor harmonic functions $\mathcal{T}_{i j}^{n l m}$ and $\mathcal{S}_{i j}^{n l m}$ (defined in appendix A), which form a complete basis on $\mathbb{S}^{3}[33,35,36]$ :

$$
\begin{aligned}
\delta \gamma_{i j} & =a^{2} 6 \sqrt{\mathcal{V}_{o}} \sum_{n=2}^{\infty} \sum_{l=0}^{n-1} \sum_{m=-l}^{l}\left(\gamma_{n l m}^{(1)} \mathcal{S}_{i j}^{n l m}+\gamma_{n l m}^{(2)} \mathcal{T}_{i j}^{n l m}\right) \\
\delta \phi & =\sum_{n=2}^{\infty} \sum_{l=0}^{n-1} \sum_{m=-l}^{l} \delta \phi^{n l m} Q_{n l m} .
\end{aligned}
$$

Note that the sum starts at $n=2$ since the $n=1$ mode is homogeneous and thus part of the background. These harmonics are scalar eigenfunctions of the Laplacian operator on $\mathbb{S}^{3}$ : $\nabla^{2} Q_{n l m}=-\left(n^{2}-1\right) Q_{n l m}$ with $\nabla$ the covariant derivative compatible with $\Omega_{i j}$. Their explicit form is

$$
Q_{n l m}(\chi, \theta, \varphi)=\Phi_{n}^{l}(\chi) Y_{l m}(\theta, \varphi),
$$

where $Y_{l m}$ are the spherical harmonics and the expression for the 'radial' function $\Phi_{n}^{l}$ can be found in appendix A. This is analogous to the flat model, in which any scalar function can also

\footnotetext{
${ }^{3}$ Just as in the flat Universes, the scalar, vector and tensor modes decouple at first order and evolve independently of one another. In this paper, we only consider scalar perturbations.
} 
be expanded in terms of the eigenfunctions of the flat space Laplacian with eigenvalue $-k^{2}$. We will denote the flat space eigenfunctions by $Q_{k l m}$. The structure of the eigenfunctions $Q_{k l m}$ is similar to eq. (2.13), where the radial function only depends on $l$ and is simply the spherical Bessel function $j_{l}(k r)$. The curved $\Phi_{n}^{l}(\chi)$ tends to $j_{l}(k r)$ (with $n \chi \rightarrow k r$ ) in the limit of large $n$ and $\chi \ll 1$, which is exactly the limit from the closed to flat FLRW model. In this limit, the hyperspherical harmonics of the closed model $Q_{n l m}$ reduce to the spherical harmonics of the flat model $Q_{k l m}$. By comparing the eigenvalues of the physical Laplacian in both cases today, we find that:

$$
\frac{n^{2}-1}{r_{o}^{2}} \rightarrow k^{2}
$$

where $r_{o}$ is the radius of the closed Universe today and $k$ is the comoving wavenumber in the flat limit. The factor $n^{2}-1$ is typically interpreted as the curved version of $k^{2}$. In fact, it is convenient to introduce $k$ even in a closed Universe - where plane waves cannot be defined using the expression above, in order to have an instantaneous notion of wavelength in terms of the labels $n$ [14]. The radius $r_{o}$ depends on the parameters $\Omega_{\mathrm{k}}$ and $H_{0}$ as:

$$
r_{o}=\frac{1}{\sqrt{\left|\Omega_{\mathrm{k}}\right|} H_{0}} .
$$

Using $\Omega_{\mathrm{k}}=-0.005$ and Planck's estimate for the Hubble parameter $H_{0}=67.3 \mathrm{~km} \mathrm{~s}^{-1} \mathrm{Mpc}^{-1}$ we find that $r_{o} \approx 6.3 \times 10^{4} \mathrm{Mpc}$. For such a Universe, the Planck comoving pivot scale $k=0.05 \mathrm{Mpc}^{-1}$ corresponds to $n=3152$, and $k=0.002 \mathrm{Mpc}^{-1}$ corresponds to $n=126$.

As in the flat model, the first order scalar perturbations $\left(\gamma^{(1)}, \gamma^{(2)}, \delta \phi\right)$ are gauge dependent quantities, and only certain combinations of them are gauge-invariant. The gauge-invariant variable $q$ was obtained using methods developed for first-class Hamiltonian constrained systems [43], which were applied to the cosmological setting by Langlois [33]. These methods allow for the determination of a singular transformation from the constraint surface in phase space to the reduced phase space of gauge-invariant, physical degrees of freedom. The procedure is as follows. First, general relativity is cast in the Hamiltonian formalism. The phase space relevant for cosmological perturbation theory is $\Gamma_{o} \times \Gamma_{1}$, with $\Gamma_{o}$ the 4-dimensional phase space of the unperturbed background, homogeneous fields and $\Gamma_{1}$ phase space of the first order, purely inhomogeneous perturbations $\left(\gamma^{(1)}, \gamma^{(2)}, \delta \phi\right)$ and their conjugate momenta with six scalar degrees of freedom. Next, the first order Hamiltonian and momentum constraints select a surface $\Gamma_{1}^{c} \subset \Gamma_{1}$ with four (scalar) degrees of freedom. These constraints, being first class, also generate gauge transformations within $\Gamma_{1}^{c}$, which further reduce the number of degrees of freedom to two, i.e. a single gauge-invariant canonical pair $(q, p) \in \tilde{\Gamma}_{1} \subset \Gamma_{1}^{c}$. To obtain the gauge-invariant perturbation, one needs to use the first order Hamiltonian and momentum constraints to go from $\Gamma_{1}$ to the reduced phase space $\tilde{\Gamma}_{1}$, defined as the set of gauge orbits in the constraint surface $\Gamma_{1}^{c}$. This is done using Hamiltonian-Jacobi inspired equations, which select a generating function that nicely implements a singular coordinate transformation from $\Gamma_{1}^{c}$ to $\tilde{\Gamma}_{1}$. As a result one obtains the following expression for the gauge-invariant variable $q[33]$ :

$$
q_{n l m}=\delta \phi_{n l m}-\sqrt{\mathcal{V}_{0}} \frac{\dot{\phi}}{H}\left(\gamma_{n l m}^{(1)}+\gamma_{n l m}^{(2)}\right)
$$

where $q$ is written in terms of hyperspherical harmonics as $q=\sum_{n=2}^{\infty} \sum_{l=0}^{n-1} \sum_{m=-l}^{l} q_{n l m} Q_{n l m}$. The gauge-invariant variable $q$ is proportional to the usual curvature perturbation $\zeta: q=$ 
$(\dot{\phi} / H) \zeta{ }^{4}$ The dynamics of $q$ on the reduced phase space is then generated by the second order Hamiltonian constraint in which only terms quadratic in the first order perturbations are kept. This yields a total quadratic Hamiltonian which is then used to obtain the following equation of motion for $q_{n l m}$ :

$$
\ddot{q}_{n l m}+b(n, t) \dot{q}_{n l m}+c(n, t) q_{n l m}=0
$$

where for each mode $b$ and $c$ are completely determined by the background evolution:

$$
\begin{aligned}
& b(n, t)=3 H+\frac{32 \pi G a^{3} \dot{a} \dot{\phi} V^{\prime}(\phi)+48 \pi G a^{2} \dot{a}^{2} \dot{\phi}^{2}-8 \pi G a^{2} \dot{\phi}^{2}\left(8 \pi G a^{2}\left(\dot{\phi}^{2}-2 V\right)+2\right)}{2 a \dot{a}\left(2\left(n^{2}-4\right) \dot{a}^{2}+8 \pi G a^{2} \dot{\phi}^{2}\right)} \\
& c(n, t)=\frac{8 \pi G}{a^{2} \dot{a}^{2}\left(2\left(n^{2}-4\right) \dot{a}^{2}+8 \pi G a^{2} \dot{\phi}^{2}\right)} \\
& {\left[\begin{array}{c}
\frac{\dot{a}^{4}\left(n^{2}-4\right)\left(n^{2}-1+a^{2} V^{\prime \prime}\right)}{4 \pi G}+\left(4 n^{2}-7\right) a^{3} \dot{a}^{3} \dot{\phi} V^{\prime}-\pi G \frac{n^{2}-1}{n^{2}-4} a^{4} \dot{\phi}^{4}\left[8 \pi G a^{2}\left(\dot{\phi}^{2}+2 V\right)-6\right] \\
+\left(n^{2}-1\right) a^{2} \dot{a}^{2}\left(-6 \pi G \frac{n^{2}-5}{n^{2}-4} a^{2} \dot{\phi}^{4}+4 \pi G a^{2} \dot{\phi}^{2} V+\frac{3}{2} \dot{\phi}^{2}+\frac{9}{2} \dot{a}^{2} \dot{\phi}^{2}\right) \\
\left.\quad+a^{3} \dot{a}\left[a \dot{a} \dot{\phi}^{2} V^{\prime \prime}+2 a \dot{a} V^{\prime 2}+4 \pi G a^{2} \dot{\phi} V^{\prime}\left(\dot{\phi}^{2}+2 V\right)-\dot{\phi} V^{\prime}\right]\right]
\end{array}\right.}
\end{aligned}
$$

with a prime denoting a derivatives with respect to $\phi$. A few comments are in order concerning the evolution of $q^{\text {nlm }}$ :

- Given an inflationary potential and proper initial conditions chosen at some initial time, eq. (2.17) describes the evolution of $q_{n l m}$ all the way till the end of inflation.

- It is immediately obvious that the equation of motion decouples for each mode, which allows one to solve the evolution of the perturbations mode by mode. This is not surprising as the same is true in the absence of spatial curvature.

- In the limit of large radius $r_{o}$ and large $n,(2.17)$ reduces to the familiar evolution equation in the case of flat spatial curvature [42]. Moreover, the divergent behavior for $n=2$ in (2.17) is not a problem, because the $n=2$ mode for $q$ vanishes identically.

- If the background is given by a de Sitter geometry, then $b(n, t)=3 H$ and $c(n, t)=$ $-a^{-2}\left(n^{2}-1\right)$.

Having the equations of motion for both the background and gauge-invariant linear perturbations, we are set to study their evolution during inflation. Let us now discuss the choice of initial conditions for the background and perturbations. ${ }^{5}$

\footnotetext{
${ }^{4}$ In terms of the scalar field perturbation $\delta \phi$ and Bardeen potential $\Psi$, this gauge-invariant quantity is $q=$ $\delta \phi+\frac{\dot{\phi}}{H} \Psi[42]$.

${ }^{5}$ In the post inflationary phase, during the radiation and matter domination era, the evolution of cosmological perturbations is governed by the Boltzmann equations which is implemented in the publicly available code CAMB [14].
} 


\section{Initial conditions}

The dynamics of the background during the inflationary era is described by Eqs. (2.3)-(2.6). The initial data required for solving this system of evolution equations consists of a choice of initial conditions $\left(a_{0}, \phi_{0}, \dot{\phi}_{0}\right)$. In addition, one must fix the value of the mass parameter in the potential $V(\phi)$ of the inflaton. In our analysis, we have considered a space of initial data and inflaton masses restricted by the condition that background solutions must include an inflationary regime long enough so that all observable modes in the CMB are within the comoving Hubble horizon at the onset of the inflation. The onset of inflation $t_{0}$ is defined as the instant at which $\ddot{a}\left(t_{0}\right)=0$ and the comoving Hubble horizon assumes its maximal value. Explicitly, we adopt the condition:

$$
\lambda_{\max } \lesssim \frac{r_{o}}{a\left(t_{0}\right) H\left(t_{0}\right)}
$$

where $\lambda_{\max }$ is the comoving wavelength of the largest observable mode. Let us describe the procedure adopted for implementing this condition.

Consider first the case of a quadratic potential (2.7), for definiteness. In order to fix the mass parameter, we first assume that: (i) the Universe was approximately flat at the time $t_{*}$ when $k_{*}=0.05 \mathrm{Mpc}^{-1}$ exited the horizon, and (ii) the slow-roll approximation is valid at $t_{*}$. Under these approximations, we can use the usual expressions for the amplitude of the scalar power spectrum $A_{\mathrm{s}}$ and its running $n_{\mathrm{s}}$ in terms of background quantities to obtain:

$$
\epsilon=\frac{1-n_{\mathrm{s}}}{4}, \quad H_{*}=\sqrt{\frac{\pi A_{\mathrm{s}} \epsilon}{G}}
$$

where $\epsilon=-\dot{H} / H^{2}$ is the first slow-roll parameter. The scale factor at horizon crossing is by definition equal to $a_{*} / r_{o}=k_{*} / H_{*}$, leading to a formula for $a_{*}$ in terms of the observable quantities $A_{\mathrm{s}}$ and $n_{\mathrm{s}}$ :

$$
a_{*}=2 r_{o} k_{*} \sqrt{\frac{G}{\pi A_{\mathrm{S}}\left(1-n_{\mathrm{s}}\right)}} .
$$

Moreover, the slow-roll parameter is related to the field configuration by

$$
\dot{\phi}_{*}^{2}(\epsilon-3)+\epsilon m^{2} \phi_{*}^{2}=0,
$$

and, under the assumed approximations, the background dynamics is described by:

$$
\begin{gathered}
3 H \dot{\phi}+m^{2} \phi=0, \\
H^{2}=\frac{8 \pi G}{3}\left(\frac{\dot{\phi}^{2}}{2}+\frac{m^{2} \phi^{2}}{2}\right) .
\end{gathered}
$$

Combining Eqs. (3.4), (3.5) and (3.6), we can solve for the mass and the field configuration at $t_{*}$ :

$$
\phi_{*}=\frac{-3+\left(1-n_{\mathrm{s}}\right) / 4}{3 \sqrt{\pi G\left(1-n_{\mathrm{s}}\right)}}, \quad \dot{\phi}_{*}=\frac{\sqrt{A_{\mathrm{s}}}\left(1-n_{\mathrm{s}}\right)}{8 G}, \quad m=\frac{3}{2} \frac{\left(1-n_{\mathrm{s}}\right) \sqrt{\pi A_{\mathrm{s}}}}{\sqrt{G\left(11+n_{\mathrm{s}}\right)}},
$$

Allowing the parameters $A_{\mathrm{s}}$ and $n_{\mathrm{s}}$ to vary within the $2 \sigma$ region determined by the Planck analysis, we obtain a window $\mathcal{I}=\left\{\left(a_{*}, \phi_{*}, \dot{\phi}_{*}\right), m\right\}$ of initial data and inflaton masses $m$ compatible with the observed data. Each point in this space of allowed initial conditions determines 
a specific evolution of the background, for which the condition (3.1) can be explicitly checked. Our analysis is based on background solutions in the observationally selected window $\mathcal{I}$ :

$$
\begin{gathered}
\phi_{*}=2.99 \pm 0.52 m_{\mathrm{Pl}}, \quad \dot{\phi}_{*}=(-2.08 \pm 0.73) \times 10^{-7} m_{\mathrm{Pl}}^{2}, \\
a_{*}=(3.3 \pm 0.6) \times 10^{-54} r_{o}, \quad m=(1.28 \pm 0.45) \times 10^{-6} m_{\mathrm{Pl}},
\end{gathered}
$$

such that the condition (3.1) is satisfied, where $m_{\mathrm{Pl}}=\sqrt{\hbar c / G}$ is the Planck mass.

The assumptions (i) and (ii) can be explicitly verified for solutions in $\mathcal{I}$. In order to do so, we first compare the curvature term in the Friedmann equation (2.3) to the value of the Hubble parameter at the horizon crossing time $t_{*}$ using Eqs. (3.2) and (3.3). For $\Omega_{\mathrm{k}}=-0.005$, and taking the best estimates for $A_{\mathrm{s}}$ and $n_{\mathrm{s}}$ from Planck [6], we find:

$$
\left|\Omega_{\mathrm{k}}\right| \frac{a_{0}^{2} H_{0}^{2}}{a_{*}^{2} H_{*}^{2}} \simeq 8 \times 10^{-13},
$$

which shows that the flat approximation is indeed valid at $t_{*}$. In addition, the interval $n_{\mathrm{s}} \pm$ $2 \sigma_{n_{\mathrm{s}}}$ provided by Planck corresponds to $\epsilon \in(0.006,0.012)$, which is consistent with a slow-roll approximation, showing that assumption (ii) is also valid.

For a given background solution $a(t), \phi(t)$ selected in this manner, we fix initial conditions for the evolution of the gauge-invariant perturbations $q_{n l m}(t)$ at the onset of inflation $t_{0}$. The perturbations are quantized with the application of standard techniques, and the initial state of the quantized perturbations is chosen to be the instantaneous vacuum at $t_{0}$, defined - as in the flat case - as the ground state of the instantaneous Hamiltonian $H\left(t_{0}\right)$ [44, 45], in the static limit. Such state can be determined as follows. The equations of motion for the perturbations, Eq. (2.17), when written for the Mukhanov-Sasaki variable $v=a q$ and in terms of the conformal time $\eta$, reduce in the static limit to:

$$
v_{n l m}^{\prime \prime}+\left[m^{2} a^{2}\left(1+\frac{6}{n^{2}-4}\right)+\left(n^{2}-1\right)\right] v_{n l m}=0 .
$$

Here, the primes represent derivatives with respect to the conformal time $\eta$. These equations describe a set of harmonic oscillators with mode-dependent frequencies

$$
\omega_{n}=\sqrt{m^{2} a^{2}\left(1+\frac{6}{n^{2}-4}\right)+\left(n^{2}-1\right)} .
$$

Recall that a choice of vacuum state for the quantized field $q$ is equivalent to a choice of positive frequency solutions $v_{n l m}(\eta)$. In a static FLRW Universe, the vacuum state can be uniquely selected by symmetry requirements: there is a unique regular state invariant under all spatial symmetries and time-translations. Such state is the tensor product of the vacua of each of the normal modes, and is described by positive frequency solutions satisfying:

$$
v_{n l m}\left(t_{0}\right)=\frac{1}{\sqrt{2 \omega_{n}}}, \quad v_{n l m}^{\prime}\left(t_{0}\right)=-i \sqrt{\frac{\omega_{n}}{2}} .
$$

These conditions correspond to:

$$
\begin{aligned}
q_{n l m}\left(t_{0}\right) & =\frac{1}{a_{0} \sqrt{2 \omega_{n}}}, \\
\dot{q}_{n l m}\left(t_{0}\right) & =-\frac{\dot{a}_{0}}{a_{0}^{2}} \frac{1}{\sqrt{2 \omega_{n}}}-\frac{i}{a_{0}^{2}} \sqrt{\frac{\omega_{n}}{2}} .
\end{aligned}
$$




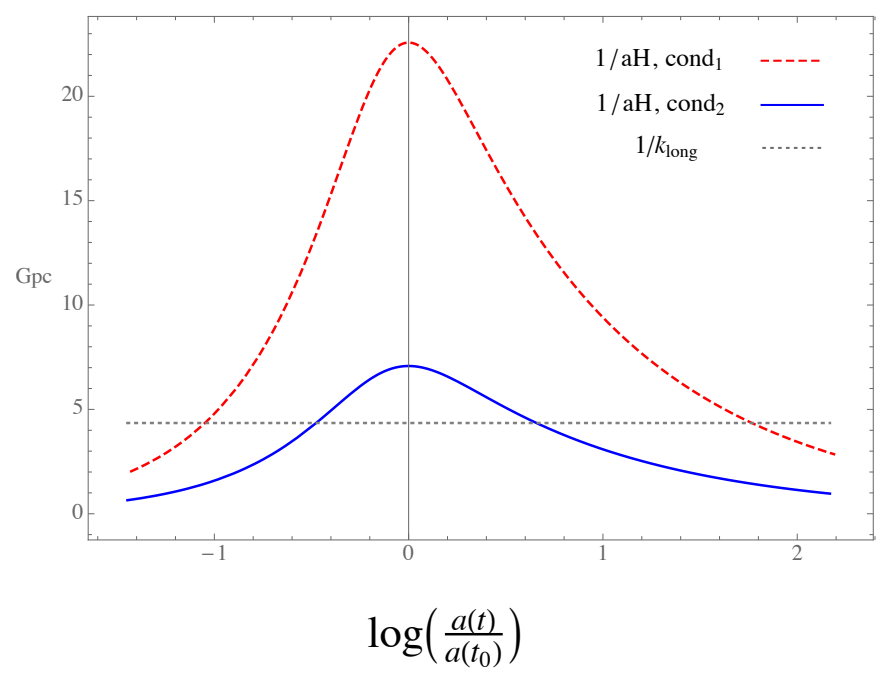

Figure 1. Comoving Hubble horizon near the onset of inflation for $\Omega_{k}=-0.005$ with two different sets of initial conditions in the space of allowed initial data. The dashed (red) curve represents a typical solution, with the the largest observable mode $k_{\text {long }}$ well within the horizon at the onset of inflation $t_{0}$. The solid (blue) curve represents a limiting case, with $1 / k_{\text {long }}$ close to the comoving Hubble horizon at $t_{0}$, for which the effects of the spatial curvature are maximal.

In a dynamical background, the instantaneous vacuum at $t_{0}$ is defined as the vacuum associated with normal modes satisfying (3.14), with frequencies $\omega_{n}$ given by Eq. (3.12) for the instantaneous scale factor $a\left(t_{0}\right)$.

We evolve the initial conditions (3.14) for a family of background solutions $(a(t), \phi(t))$ satisfying the condition (3.1). In order to estimate the wavelength of the largest observable mode $\lambda_{\text {long }}$, we set $\lambda_{\text {long }}=d_{\mathrm{CMB}}$, where $d_{\mathrm{CMB}}$ is the comoving diameter of the CMB sphere, yielding $k_{\text {long }}=2.3 \times 10^{-4} \mathrm{Mpc}^{-1}$. From Eq. (2.14), this corresponds to $n_{\text {long }} \sim 14$. For such solutions, all observable modes are within the comoving Hubble horizon at the onset of inflation $t_{0}$. In Fig. 1, we show the evolution of the comoving Hubble horizon around the onset of inflation for two distinct backgrounds and compare it with $1 / k_{\text {long. }}$. The solution 'cond 1 ' represents the case for which the initial conditions for the background geometry are provided using the best fit values of $A_{s}$ and $n_{s}$. For this case the largest observable mode is well within the horizon at $t_{0}$. The variation of the frequency term in the full Mukhanov-Sasaki equation is then checked to be negligible around $t_{0}$, with $H \dot{w}_{n} / \omega_{n} \ll 1$ for all observable modes $n>14$. The solution ' cond $_{2}$ ' represents the extreme case considered in our analysis. In this case, $1 / k_{\text {long }}$ is close enough to the horizon so that the fractional variation of the frequency becomes of the order of $10 \%$ during the few tenths of an e-fold before $t_{0}$ while $k_{\text {long }}$ is within the horizon. This extreme case marks the point in the parameter space for which the static approximation starts becoming unreliable. In the next section, these two conditions illustrate the range of possible effects on the primordial power spectrum and observed anisotropies in the CMB due to spatial curvature when $\Omega_{\mathrm{k}}=-0.005$.

The case of the Starobinsky potential is treated similarly. The mass parameter $M$ in the potential (2.8) is fixed by taking a flat slow-roll approximation at horizon crossing for the mode $k_{*}$ and using the number of $e$-folds $\left(N_{*}\right)$ between the horizon crossing and the end of inflation. This leads to formulas analogous to eqs. (3.3) and (3.7) which give (see [46] for details of estimation of parameters for the Starobinsky potential): 


$$
\begin{gathered}
\phi_{*}=1.08 \pm 0.02 m_{\mathrm{Pl}}, \quad \dot{\phi}_{*}=(-4.80 \pm 0.70) \times 10^{-9} m_{\mathrm{Pl}}^{2}, \\
a_{*}=2.11 \pm 0.14 \times 10^{-53} r_{o}, \quad M=(2.56 \pm 0.18) \times 10^{-6} m_{\mathrm{Pl}}
\end{gathered}
$$

The initial conditions for the perturbations are also set at the onset of inflation by choosing the instantaneous vacuum determined by the conditions (3.14).

\section{Results}

Given the initial data for the background and the gauge-invariant perturbations, we numerically evolve the equations of motion of the background and the quantum perturbations. This section is divided into two subsections. In the first, we discuss the evolution of the background spacetime and show that the presence of spatial curvature can affect the inflationary dynamics and potentially leave observable imprints on the long wavelength modes. The second subsection describes the evolution of scalar perturbations, their power spectrum at the end of inflation and the resulting temperature anisotropy spectrum $C_{\ell}$ observed in the CMB.

\subsection{Why does spatial curvature matter?}

One of the attractive features of the inflationary paradigm is that it solves the flatness problem. Starting from a generic initial condition inflation dilutes away all spatial curvature effects that might have been present before the onset on inflation. As result at the end of inflation and today the Universe is extremely close to being spatially flat: $\left|\Omega_{\mathrm{k}}\right|<0.005$, i.e., the contribution of spatial curvature to the total energy density of our Universe today is less than $0.5 \%$. What about in the past? The post inflationary evolution of the Universe is described by the $\Lambda$ CDM model and the associated Friedmann equation is given by eq. (2.9). The fraction of energy density due to the spatial curvature decreases monotonically towards the past. Using the current estimates of the cosmological parameters $\Omega_{\mathrm{m}}, \Omega_{\mathrm{r}}, \Omega_{\Lambda}$ and $\Omega_{\mathrm{k}}$ from Planck, it is evident from eq. (2.9) that at the beginning of the radiation dominated era (approximately $66 e$-folds before today), which — assuming instantaneous reheating — is the same as the end of inflation:

$$
\left.\left.\frac{\rho_{\mathrm{k}}}{\rho_{\mathrm{r}}}\right|_{\text {beg rad }} \approx 10^{-57} \approx \frac{\rho_{\mathrm{k}}}{\rho_{\phi}}\right|_{\text {end inf }},
$$

where $\rho_{\mathrm{k}}=\frac{3}{8 \pi G} \frac{\Omega_{\mathrm{k}} H_{0}^{2}}{a^{2} / a_{0}^{2}}, \rho_{\mathrm{r}}=\frac{3}{8 \pi G} \frac{\Omega_{\mathrm{r}} H_{0}^{2}}{a^{4} / a_{0}^{4}}$ and $\rho_{\phi}=\dot{\phi}^{2} / 2+V(\phi)$ is the energy density of the inflaton. This shows that the contribution of spatial curvature at the beginning of the radiation era is $10^{-55 \%}$. Hence, the effect of spatial curvature has been practically insignificant starting from the end of inflation till today.

During inflation, however, the spacetime is quasi-de Sitter and $\rho_{\phi}$ remains nearly constant while $\rho_{\mathrm{k}}$ scales as $a^{-2}$. In the backward evolution the spatial curvature terms keep getting stronger and approximately $60 e$-folds before the end of inflation $\rho_{\mathrm{k}}$ can become comparable to $\rho_{\phi}$, potentially leading to the breakdown of the slow-roll phase [26-28]. Therefore, the long wavelength modes which exit the Hubble horizon during the early stages of inflation will be influenced by the spatial curvature and their power spectrum can carry the imprints of spatial curvature. This is in contrast to the short wavelength modes which exit the Hubble horizon later and thus remain unaffected by the spatial curvature. Consequently, their power spectrum is practically the same as in the absence of spatial curvature.

Fig. 2 shows the evolution of the Hubble horizon, $(a H)^{-1}$, during inflation and radiation era all the way till the CMB for $\Omega_{\mathrm{k}}<0$ and $\Omega_{\mathrm{k}}=0$. It is evident from the figure that the 


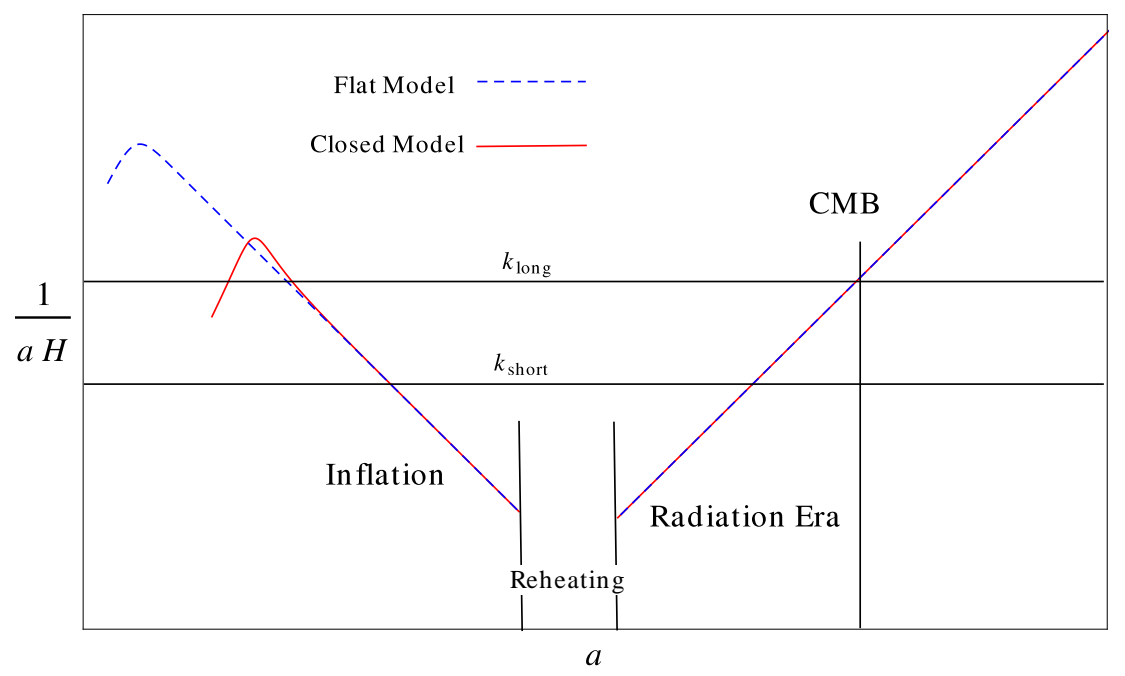

Figure 2. Comparison of Hubble horizon during and after inflation in a flat FRLW model and a closed model with $\Omega_{\mathrm{k}}=-0.1$. The difference due to spatial curvature is important close to the onset of inflation and remains negligible throughout the rest of the future evolution. This indicates that the long wavelength modes which exit the curvature radius earlier during inflation may carry imprints of the spatial curvature. These potential imprints will, for instance, affect their power spectrum.

Hubble horizons for flat and closed models differ in the early stages of inflation, while agreeing with each other extremely well in the future. This indicates that the long wavelength modes (denoted by $k_{\text {long }}$ ) which exit the horizon during the early stages of inflation may carry imprints of the spatial curvature, while the short wavelength modes (denoted by $k_{\text {short }}$ ) which exit the horizon later will remain unaffected by the spatial curvature.

Remark: Note that it is not a priori clear whether or not inflation will last long enough in the presence of spatial curvature. As pointed out in [26], the amount of inflation in the closed model is bounded above and the bound is dictated by the parameter $\Omega_{\mathrm{k}}$. As a result, the space of initial conditions leading to sufficient amount of $e$-folds in the closed model will be smaller than for the flat model. In this paper, we are interested in finding at least one set of initial conditions for which the desired inflationary phase takes place. As described in the previous section, we found a family of initial conditions on $\phi, \dot{\phi}$ and $a$ which lead to the desired inflationary phase and are compatible with observations within the error bars. A more quantitative analysis of the space of initial conditions and the viability of desired inflationary phase requires investigation of a suitable measure on the space of initial data and will be studied in detail in a quantum gravitational extension of this paradigm in [47].

Let us now study the evolution of the scalar perturbations and analyze the potentially observable imprints of spatial curvature on the CMB.

\subsection{Power spectrum}

We now consider the evolution of the gauge-invariant quantum scalar perturbations $q_{n l m}$ on the inflationary background geometry discussed above. As discussed in Sec. 3, $b(n, t)$ and $c(n, t)$, the coefficients of $\dot{q}$ and $q$ in the equation of motion for $q$ in the closed model (see eq. (2.17)), take a very different form compared to the flat FLRW model. In particular, $b(n, t)$ for the flat model is simply $3 H(t)$, whereas in the closed model $b(n, t)$ also depends on the comoving wavenumber $n$ in a rather complicated fashion. Therefore, the evolution of scalar perturbations 

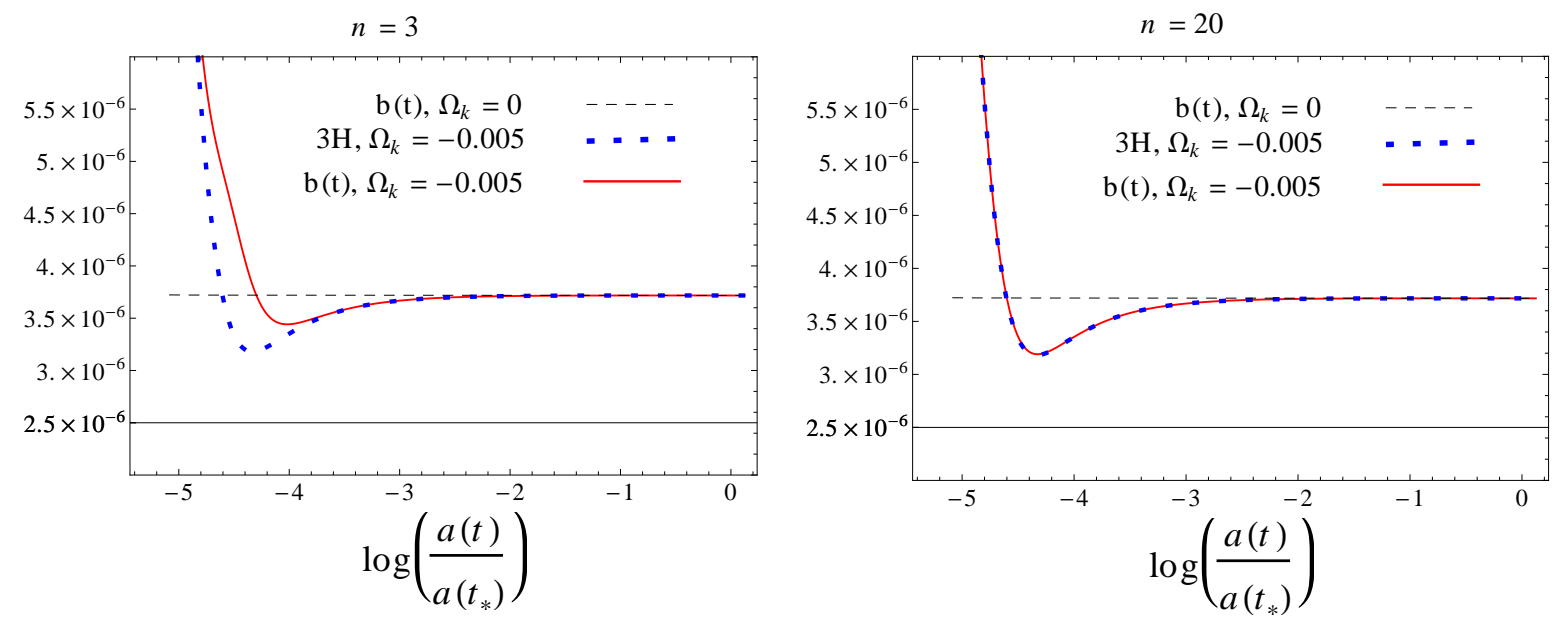

Figure 3. Comparison of the coefficient $b(n, t)$ in the evolution equation (2.17) for the gauge-invariant scalar perturbation $q_{n l m}$ in a closed FLRW model for $\Omega_{\mathrm{k}}=-0.005$ with $3 H$ in the same closed model and with $b(n, t)$ for a flat model. The left panel corresponds to the wavenumber $n=3$ for which $b(n, t)$ is distinct from $3 H$ in the closed model. The right panel corresponds to $n=20$ for which the two quantities are practically indistinguishable. In both cases, $b(n, t)$ for the flat model is different from $3 H$ and $b(n, t)$ for the closed model. These differences become more prominent for larger magnitudes of $\Omega_{\mathrm{k}}$.
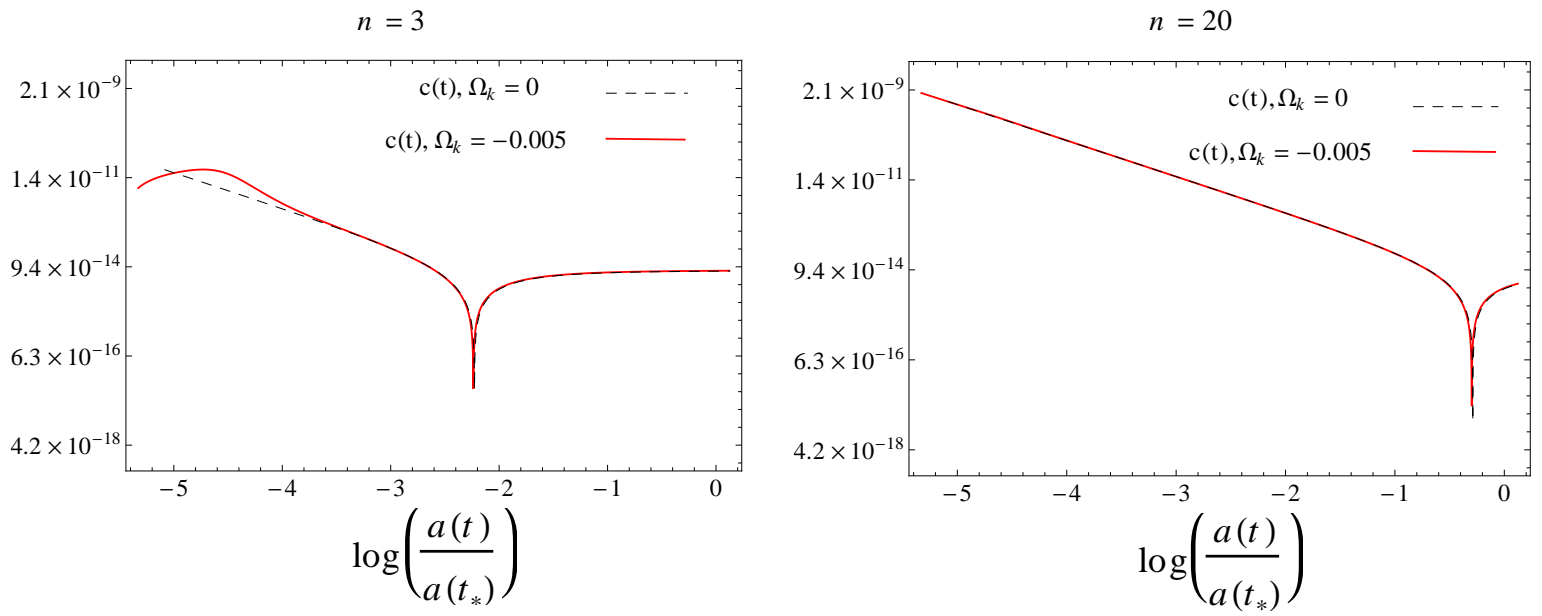

Figure 4. Comparison of the coefficient $c(n, t)$ in the evolution equation (2.17) for the gauge-invariant scalar perturbation $q_{n l m}$ in a closed FLRW model for $\Omega_{\mathrm{k}}=-0.005$ with that in a flat model. The left panel corresponds to the wavenumber $n=3$ for which $c(n, t)$ in closed model shows deviations from that in the flat model. The right panel corresponds to $n=20$ for which the two curves are practically indistinguishable.

in a closed inflationary model is different from the flat model in two ways. First, the evolution of background quantities $H(t)$ and $a(t)$ are different which leads to differences in the causal horizon (fig. 2) and second, the evolution equation for the perturbations acquires additional $n$ dependence for the closed model whose numerical effect becomes more important for small $n$. Fig. 3 and 4 respectively show the evolution of the coefficients $b(n, t)$ and $c(n, t)$ in eq. $(2.17)$ 


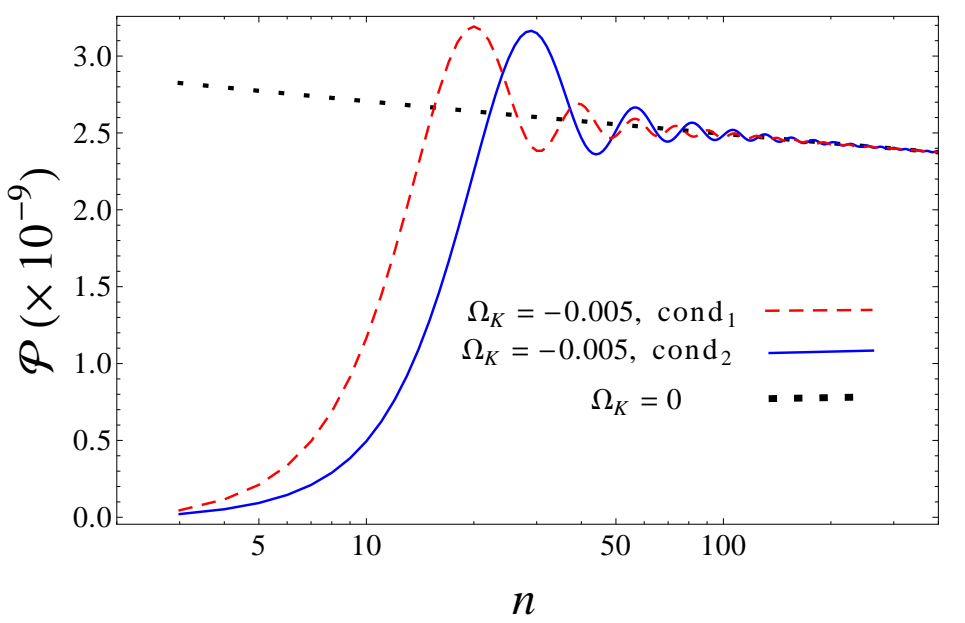

Figure 5. Scalar power spectra for the closed model for $\Omega_{\mathrm{k}}=-0.005$ with two different sets of initial conditions for the background geometry. The dashed (red) curve corresponds to: $\phi\left(t_{*}\right)=3.07 \mathrm{~m}_{\mathrm{Pl}}$, $\dot{\phi}\left(t_{*}\right)=-2.08 \times 10^{-7} m_{\mathrm{Pl}}^{2}$ and the solid (blue) curve corresponds to: $\phi\left(t_{*}\right)=2.99 m_{\mathrm{Pl}}, \dot{\phi}\left(t_{*}\right)=$ $-2.13 \times 10^{-7} m_{\mathrm{Pl}}^{2}$. The dotted straight curve shows the almost scale-invariant spectrum. The closed model power spectrum shows oscillations for small $n$ and approaches the flat power spectrum for large $n$. Interestingly, the average power in the small $n$ regime is suppressed compared to the usual nearly scale-invariant power spectrum. Here we have shown the power spectrum for $3 \leq n \leq 400$. However, for $\Omega_{\mathrm{k}}=-0.005$, only the modes with $n \gtrsim 14$ are observable.

for the closed model compared with that in the flat model during inflation. The horizontal axes in both plots show the number of $e$-folds from the time $t_{*}$ when the reference mode $k_{*}=0.002 \mathrm{Mpc}^{-1}$ exits the Hubble horizon. It is apparent from the figures that approximately $4 e$-folds before the horizon exit:

- $b(n, t)$ for the closed model is different from that in the flat model for both $n=3$ and $n=20$ (fig. 3),

- for large $n, b(n, t)$ in the closed model approaches $3 H$ (right panel of fig. 3 ) and

- $c(n, t)$ in the closed model shows deviations from that in the flat model for small $n$ (e.g., $n=3$ shown in the left panel of fig. 4) while for large $n$ they are practically indistinguishable (e.g., $n=20$ shown in the right panel of fig. 4 ).

Therefore, the spatial curvature effects are more prominent during the early stages of inflation and for small $n$ modes which exit the Hubble horizon at these times.

Recall that in the flat model the initial conditions for the perturbations are given a few $e$-folds before the mode with the longest wavelength exits the curvature radius and the scalar perturbations are assumed to be in a Bunch-Davies vacuum state as the background geometry behaves like a quasi-de Sitter spacetime. In contrast, for the closed model, the spacetime shows deviations from a quasi-de Sitter spacetime at the onset of inflation as shown in fig. 2 . Consequently, the Bunch-Davies approximation is violated. However, since all the observable modes are still inside the curvature radius, we provide the initial conditions assuming static initial conditions as described in Sec. 3. 
We explore all observationally compatible initial conditions for the background geometry for the two potentials considered: the quadratic potential (see eq. (3.9)) and Starobinsky potential (see (3.16)). In fig. 5 we show scalar power spectra for the two initial conditions discussed in section 3 for the quadratic potential with $\Omega_{\mathrm{k}}=-0.005$. The initial conditions are chosen such that: (i) 'cond ${ }_{1}^{\prime}$ (red, dashed curve) for which $\phi\left(t_{*}\right)=3.07 m_{\mathrm{Pl}}, \dot{\phi}\left(t_{*}\right)=-2.08 \times 10^{-7} m_{\mathrm{Pl}}^{2}$ at the time $t_{*}$ when the reference scale $k_{*}=0.002 \mathrm{Mpc}^{-1}$ exits the curvature radius, and (ii) ' $\operatorname{cond}_{2}$ ' (blue, solid curve) for which $\phi\left(t_{*}\right)=2.99 m_{\mathrm{Pl}}, \dot{\phi}\left(t_{*}\right)=-2.13 \times 10^{-7} m_{\mathrm{Pl}}^{2}$ at the same time $t_{*}$. The usual nearly scale-invariant spectrum is shown by the black dashed straight line. The power spectra for the closed model have two main features. First, the closed model power spectrum is the same as that in the flat model for large $n$. These modes correspond to short wavelength modes that exit the Hubble horizon later during inflation. Second, for small $n$ (corresponding to longer wavelength modes which exit the Hubble horizon earlier during inflation) the closed model power spectrum oscillates around the flat one. Interestingly, these oscillations when averaged over a range of $n$ show that: $\left(\mathcal{P}_{\text {closed }}\right)_{\text {avg }}<\left(\mathcal{P}_{\text {flat }}\right)_{\text {avg. }}$. That is, the closed model power spectrum has a power deficit at large scales compared to the flat model. Numerical computations with the Starobinsky potential give rise to similar results. Note that this may not be true for the tensor perturbations, because heuristically similarly to the flat case where the tensor-to-scalar ratio is different for the two potentials while the scalar power spectrum is the same [1].

The question now is: are these features strong enough to have observational imprints on the temperature anisotropy spectrum observed in the CMB? For instance, do these oscillations show up in the temperature anisotropy spectrum of the CMB $\left(C_{\ell}^{\mathrm{TT}}\right)$ observed today and more importantly, does the deficit of power in the power spectrum at the end of inflation lead to a suppression of power in the $\mathrm{CMB}$, which is one of the large scale anomalies reported by the recent Planck and WMAP missions?

\subsection{Temperature anisotropy spectrum}

To answer these questions, we evolve the scalar power spectrum at the end of inflation using the Boltzmann code CAMB to compute the temperature anisotropy spectrum $C_{\ell}^{\mathrm{TT}}$ at the surface of last scattering [14]. Fig. 6 shows the resulting temperature anisotropy power spectrum for a closed model with $\Omega_{\mathrm{k}}=-0.005$ with initial conditions corresponding to the power spectra in fig. 5. The dashed (red) curve corresponds to $\phi\left(t_{*}\right)=3.07 m_{\mathrm{Pl}}, \dot{\phi}\left(t_{*}\right)=-2.08 \times 10^{-7} \mathrm{~m}_{\mathrm{Pl}}^{2}$ (denoted as cond 1 in the figure) and the solid (blue) one corresponds to $\phi\left(t_{*}\right)=2.99 \mathrm{~m}_{\mathrm{Pl}}$, $\dot{\phi}\left(t_{*}\right)=-2.13 \times 10^{-7} m_{\mathrm{Pl}}^{2}$ (denoted as cond 2 in the figure). It is evident from the figure that the closed model $C_{\ell}$ agrees extremely well with the Planck data and the predictions of the standard inflationary scenario with flat FLRW model at large $\ell$. Interestingly, for $\ell \lesssim 20$, however, the closed model shows deficit of power as compared to the flat model as expected from fig. 5. The second, more extreme condition has less number of $e$-folds during inflation compared to the first condition, leading to suppression as large as $\sim 30 \%$ in $C_{\ell}^{\mathrm{TT}}$ at $\ell=2$, while the first condition leads to $\sim 10 \%$ suppression there. The corresponding E-mode polarization spectrum and its cross correlation with the temperature spectrum are shown in fig. 7 , which show small deviations from the flat model at similar scales. The suppression in power is limited to the multipoles $\ell<20$, and therefore is not enough to explain the power suppression anomaly observed in the CMB. Nevertheless, as is evident from our analysis, it can contribute to the suppression of power at these scales.

Here for the concreteness of the discussion we have shown the plots for $\Omega_{\mathrm{k}}=-0.005$ which is the bound on spatial curvature given by combining Planck and BAO data. If one 


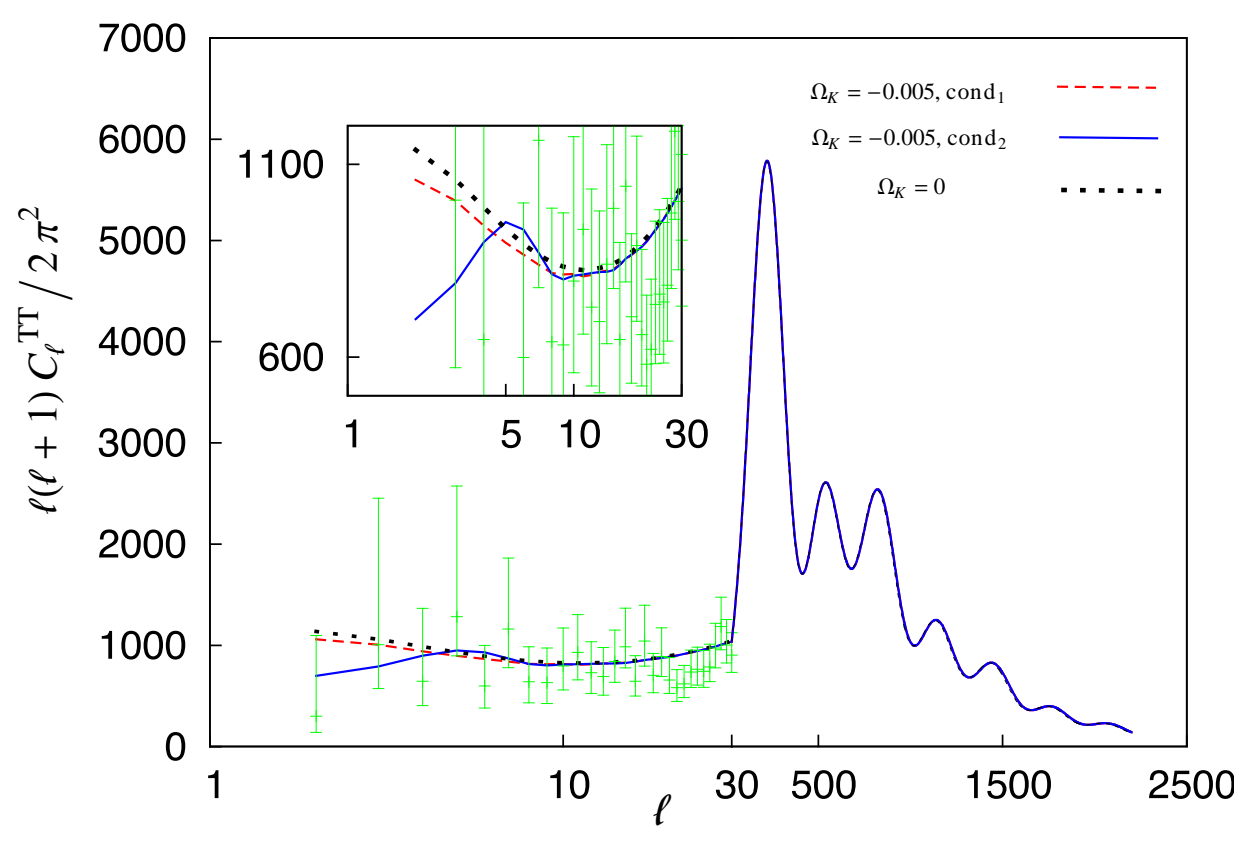

Figure 6. The temperature anisotropy power spectrum for closed (dashed and solid curves) and flat (dotted) models and the Planck 2015 data with error bars. The dashed (red) curve corresponds to: $\phi\left(t_{*}\right)=3.07 m_{\mathrm{Pl}}, \dot{\phi}\left(t_{*}\right)=-2.08 \times 10^{-7} m_{\mathrm{Pl}}^{2}$. The solid (blue) curve corresponds to : $\phi\left(t_{*}\right)=2.99 m_{\mathrm{Pl}}$, $\dot{\phi}\left(t_{*}\right)=-2.13 \times 10^{-7} m_{\mathrm{Pl}}^{2}$, both with $\Omega_{\mathrm{k}}=-0.005$. It is evident that in both cases the closed model shows deficit power at low $\ell$ 's compared to the flat model at $\ell \lesssim 10$.
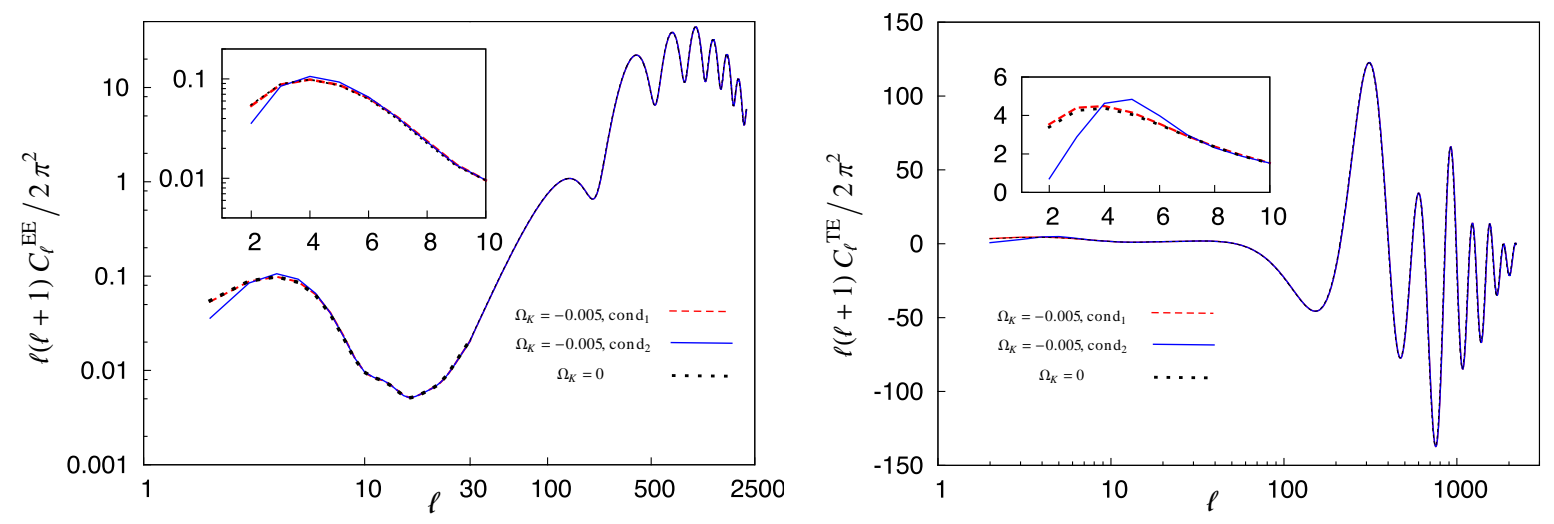

Figure 7. E-mode polarization spectrum and cross correlation with the temperature anisotropy spectrum for the closed model with the same initial conditions as in fig. 6 . There are tiny differences between the closed model and the flat model polarization spectrum at $\ell<10$.

takes a higher magnitude of $\Omega_{\mathrm{k}}$ the power suppression will be stronger, and conversely, smaller $\left|\Omega_{\mathrm{k}}\right|$ will show less power suppression. In order to find the best fit value of $\Omega_{\mathrm{k}}$ using the modified primordial power spectrum obtained here, we revisit the the estimation of cosmological parameters by comparing the resulting $C_{\ell}^{\mathrm{TT}}$ against the recent Planck and BAO data using the publicly available Markov Chain Monte Carlo code COSMOMC [48]. We allow variations of all 
cosmological parameters. We find that, since the modifications are limited to very low $\ell$ 's, they practically have no effect on the parameter estimation. In particular, we found: $\Omega_{\mathrm{k}}=$ $0.000 \pm 0.005$, which is exactly what is reported in [6]. Therefore, although conceptually the Planck estimation of $\Omega_{\mathrm{k}}$ in [6] is inconsistent, the estimation of cosmological parameters in the presence of spatial curvature is phenomenologically robust.

\section{Discussion}

The presence of spatial curvature can affect the CMB observations in two ways: modifications in the Boltzmann equations which affect the transfer function from the end of inflation till today and in the spectrum of primordial fluctuations at the end of inflation. The former possibility has been studied (see e.g. [14]), while the latter possibility has only been addressed using approximate methods $[29,30,32]$. However, a full treatment of the primordial fluctuations during inflation in the presence of positive spatial curvature had been missing so far. In this paper, we studied the inflationary dynamics of the closed FLRW model in the presence of the quadratic and Starobinsky potential and analyzed the evolution of the cosmological perturbations. We first obtained the gauge-invariant scalar perturbations following Hamiltonian methods described in [33] and derived the quadratic Hamiltonian that governs their evolution on the inflationary background geometry. These perturbations were then numerically evolved by providing suitable initial conditions before the onset of slow-roll. We computed the resulting power spectrum at the end of inflation, which provided the initial conditions for the Boltzmann equations that describe the evolution of the cosmological linear perturbations in the post inflationary phase. We then computed the temperature anisotropy spectrum at the surface of last scattering using CAMB [14]. Naturally, a modification in the primordial power spectrum can leave observational imprints in the temperature and polarization spectrum of the CMB and can alter the estimation of cosmological parameters.

Our analysis shows that not only the background inflationary dynamics of a closed FLRW model is different from that in the flat model, but also the scalar power spectrum at the end of inflation carries signatures of these differences. This further leads to observational imprints on the long wavelength modes observed in the CMB. We performed numerical computation of $C_{\ell}^{\mathrm{TT}}$ by exploring initial conditions for the background geometry allowed by the observational error bars. We found that the scalar power spectrum in closed model shows oscillatory behavior for long wavelength modes whose wavelengths are comparable to the size of the observable Universe today, a feature not present in the flat model. For short wavelength modes, on the other hand, there is excellent agreement with the predictions of the spatially flat inflationary spacetime. Interestingly, we find that although the oscillatory behavior of the power spectrum is diluted in the temperature power spectrum for short scales (see fig. 6), there is deficit of power at low $\ell$ 's compared to the predictions of the spatially flat model. For $\Omega_{\mathrm{k}}=-0.005$, the suppression ranges between $10-30 \%$ for $\ell=2$ depending on the initial conditions. In the limit $\Omega_{\mathrm{k}} \rightarrow 0$ the power spectrum in the flat model, i.e. without oscillations and suppression, is recovered. Since the suppression is limited to $\ell<10$, it is not enough to explain the anomaly observed by recent CMB experiments. Nonetheless, our analysis shows that these anomalies can be partially accounted for by the inclusion of positive spatial curvature (i.e. with $\Omega_{\mathrm{k}}<0$ ).

In the estimation of $\Omega_{\mathrm{k}}$ in [6], the primordial power spectrum was assumed to be nearly scale-invariant. This is conceptually inconsistent, as the spatial curvature modifies the primordial power spectrum. For $\Omega_{\mathrm{k}}=-0.005$, which is within the Planck constraints, these modifications are in the observable range. Nevertheless, since the observational effects of the 
spatial curvature are limited to very low multipoles, spatial curvature has negligible effects on the estimation of the cosmological parameters. Therefore, estimation of all cosmological parameters, including $\Omega_{\mathrm{k}}$, by Planck in [6] is phenomenologically robust under the inclusion of positive spatial curvature.

In this paper, we restricted our analysis to initial conditions compatible with observations. In order to study the naturalness and the origin of these initial conditions for inflation in the closed FLRW model, one needs to extend the analysis to the Planck scale and introduce a suitable measure on the full space of initial conditions. These questions have already been addressed in the setting of loop quantum cosmology (LQC) for the flat FLRW model in the presence of quadratic and Starobinsky potential [46, 49-51]. In a future work [47], we will use the LQC model of the closed FLRW spacetime developed in [52] to address these issues and extend the framework of quantum fields on quantum spacetime of $[53,54]$ to evolve cosmological perturbations all the way from the Planck scale to the end of inflation. Another natural extension of this work is to study the evolution of tensor modes in closed inflationary FLRW spacetime [55]. A priori, it is not clear whether the tensor modes will also show a similar suppression as the quadratic Hamiltonian for tensor modes is quite different from that of the scalar modes. Spatial curvature effects in the tensor spectrum may lead to interesting features in the B-mode polarization spectrum at large scales which may become observable in future CMB experiments. In recent works [56-58], it has been shown that coupling between long and short wavelength modes in presence of non-gaussianity in the flat model can lead to hemispherical asymmetry which is another CMB anomaly observed in the CMB. In future work, it will be interesting to study how the presence of positive spatial curvature can affect these results.

\section{Acknowledgements}

We are grateful to Abhay Ashtekar for ample discussions, guidance and suggesting this problem. We would also like to thank Eugenio Bianchi, Sarah Shandera and Donghui Jeong for discussions. This work was supported by NSF grant PHY-1505411, the Eberly research funds of Penn State and a Frymoyer Fellowship to BB. NY acknowledges support from CNPq, Brazil. This work used the Extreme Science and Engineering Discovery Environment (XSEDE), which is supported by National Science Foundation grant number ACI-1053575.

\section{A Hyperspherical harmonics and linear perturbations on $\mathbb{S}^{3}$}

In this appendix we review basic properties of hyperspherical harmonics on the unit sphere $\mathbb{S}^{3}$ and discuss the expansion of linear cosmological perturbations in general closed FLRW Universes in terms of such normal modes (for more details, see $[35,59]$ ). We choose coordinates in which the metric of $S^{3}$ is of the form:

$$
d \Omega^{2}=d \chi^{2}+\sin ^{2} \chi\left(d \theta^{2}+\sin ^{2} \theta d \varphi^{2}\right),
$$

with $\chi, \theta \in[0, \pi], \varphi \in[0,2 \pi]$. We write the metric tensor as $\Omega_{i j}$, the covariant derivative associated with $\Omega$ as $\nabla$, and the corresponding Laplace-Beltrami operator as $\nabla^{2}$.

A complete set of scalar functions on $\mathbb{S}^{3}$ is given by the hyperspherical harmonics:

$$
Q_{n l m}(\chi, \theta \varphi)=\Phi_{n}^{l}(\chi) Y_{l m}(\theta, \varphi),
$$


where the $Y_{l m}(\theta, \varphi)$ are spherical harmonics on the 2-sphere and the Fock harmonics $\Phi_{n}^{l}(\chi)$ are defined as:

$$
\Phi_{n}^{l}(\chi)=\sqrt{\frac{M_{n l}}{\sin \chi}} P_{-1 / 2+n}^{-1 / 2-l}(\cos \chi), \quad M_{n l}=\Pi_{r=0}^{l}\left(n^{2}-r^{2}\right),
$$

where $P_{-1 / 2+n}^{-1 / 2-l}$ are the associated Legendre functions. The hyperspherical harmonics are eigenfunctions of the Laplacian:

$$
\nabla^{2} Q_{n l m}=-\left(n^{2}-1\right) Q_{n l m},
$$

and satisfy the orthogonality relations:

$$
\int_{\mathbb{S}^{3}} d \Omega Q_{n l m} Q_{n^{\prime} l^{\prime} m^{\prime}}=\delta_{n n^{\prime}} \delta_{l l^{\prime}} \delta_{m m^{\prime}}
$$

We can use the scalar harmonics $Q_{n l m}$ to define a set of orthogonal vector harmonics:

$$
P_{i}^{n l m}=\frac{1}{n^{2}-1} \nabla_{i} Q_{n l m}
$$

which satisfy $\nabla^{2} P_{i}=-\left(n^{2}-3\right) P_{i}$ and are normalized according to:

$$
\int_{\mathbb{S}^{3}} d \Omega \Omega^{i j} P_{i}^{n l m} P_{j}^{n l m}=\frac{1}{n^{2}-1} .
$$

We also introduce the tensor harmonics:

$$
\begin{aligned}
\mathcal{S}_{i j}^{n l m} & =\frac{1}{3} \Omega_{i j} Q_{n l m}, \\
\mathcal{T}_{i j}^{n l m} & =\left(\frac{1}{n^{2}-1} \nabla_{i} \nabla_{j}+\frac{1}{3} \Omega_{i j}\right) Q_{n l m},
\end{aligned}
$$

satisfying $\nabla^{2} \mathcal{S}_{i j}^{n l m}=-\left(n^{2}-1\right) \mathcal{S}_{i j}^{n l m}$ and $\nabla^{2} \mathcal{T}_{i j}^{n l m}=-\left(n^{2}-7\right) \mathcal{T}_{i j}^{n l m}$. These functions are orthogonal for distinct labels $n, l, m$, and satisfy the relations:

$$
\begin{aligned}
& \int d \Omega \mathcal{S}_{i j}^{n l m} \mathcal{T}_{n l m}^{i j}=0 \\
& \int d \Omega \mathcal{S}_{i j}^{n l m} \mathcal{S}_{n l m}^{i j}=\frac{1}{3} \\
& \int d \Omega \mathcal{T}_{i j}^{n l m} \mathcal{T}_{n l m}^{i j}=\frac{2}{3} \frac{n^{2}-4}{n^{2}-1},
\end{aligned}
$$

where the sum is only over $i$ and $j$ and not over $n, l$ and $m$. The $\mathcal{T}_{i j}^{n l m}$ are traceless and it can be easily verified that:

$$
\nabla^{i} \nabla^{j} \mathcal{T}_{i j}^{n l m}=\frac{2}{3}\left(n^{2}-4\right) Q_{n l m} .
$$

As in the flat case, cosmological linear perturbations on a closed FLRW Universe can be decomposed into decoupled scalar, vector and tensor modes. For the case of scalar perturbations in the presence of a scalar field, the metric and field perturbations are described by eqs. (2.11) and (2.12), while the conjugate momenta are:

$$
\begin{aligned}
\delta \tilde{\pi}^{i j} & =\frac{1}{a^{2} 6 \sqrt{V_{o}}} \sum_{n=2}^{\infty} \sum_{l=0}^{n-1} \sum_{m=-l}^{l}\left(3 \pi_{n l m}^{(1)} \mathcal{S}_{i j}^{n l m}+\frac{3}{2} \frac{n^{2}-1}{n^{2}-4} \pi_{n l m}^{(2)} \mathcal{T}_{i j}^{n l m}\right) \sqrt{\Omega}, \\
\delta \tilde{\pi}_{\phi} & =\sqrt{\frac{4 \pi G}{3 \mathcal{V}_{o}}} \sum_{n=2}^{\infty} \sum_{l=0}^{n-1} \sum_{m=-l}^{l} \pi_{\phi}^{n l m} Q_{n l m} \sqrt{\Omega} .
\end{aligned}
$$


The coefficients in the expansion of the scalar perturbations and conjugate momenta are determined by:

$$
\begin{aligned}
\gamma_{n l m}^{(1)} & =\frac{1}{2 a^{2} \sqrt{V_{o}}} \int d \Omega \delta \gamma_{i j} \mathcal{S}_{i j}^{n l m} \\
\gamma_{n l m}^{(2)} & =\frac{1}{4 a^{2} \sqrt{V_{o}}} \frac{n^{2}-1}{n^{2}-4} \int d \Omega \delta \gamma_{i j} \mathcal{T}_{i j}^{n l m} \\
\pi_{n l m}^{(1)} & =6 \sqrt{V_{o}} a^{2} \int d \Omega \delta \tilde{\pi}^{i j} \mathcal{S}_{i j}^{n l m} \\
\pi_{n l m}^{(2)} & =6 \sqrt{V_{o}} a^{2} \int d \Omega \delta \tilde{\pi}^{i j} \mathcal{T}_{i j}^{n l m}
\end{aligned}
$$

Using the fundamental equal-time Poisson brackets $\left\{\delta \gamma_{i j}(x), \delta \tilde{\pi}^{k l}\left(x^{\prime}\right)\right\}=\delta\left(x-x^{\prime}\right) \delta_{(i}^{k} \delta_{j}^{l}$, one can derive the Poisson brackets for the amplitudes in the hyperspherical harmonics expansion:

$$
\left\{\gamma_{n l m}^{(a)}, \pi_{n^{\prime} l^{\prime} m^{\prime}}^{(b)}\right\}=\delta^{a b} \delta_{n n^{\prime}} \delta_{l l^{\prime}} \delta_{m m^{\prime}}
$$

Similarly, it follows from $\left\{\delta \phi(x), \delta \tilde{\pi}_{\phi}\left(x^{\prime}\right)\right\}=\delta\left(x-x^{\prime}\right)$ that

$$
\left\{\delta \phi^{n l m}, \pi_{\phi}^{n^{\prime} l^{\prime} m^{\prime}}\right\}=\delta_{n n^{\prime}} \delta_{l l^{\prime}} \delta_{m m^{\prime}} .
$$

\section{References}

[1] P. A. R. Ade et al. [Planck Collaboration], "Planck 2015 results. XX. Constraints on inflation," Astron. Astrophys. 594, A20 (2016) doi:10.1051/0004-6361/201525898

[2] WMAP Collaboration, G. Hinshaw et al., "Nine-Year Wilkinson Microwave Anisotropy Probe (WMAP) Observations: Cosmological Parameter Results", Astrophys. J. Suppl. 208 (2013) 19, arXiv:1212.5226.

[3] S. Weinberg, "Cosmology", Oxford University Press, Oxford, 2008.

[4] V. Mukhanov, "Physical foundations of cosmology", Cambridge University Press, 2005.

[5] S. Dodelson, "Modern Cosmology", Academic Press, Amsterdam, 2003.

[6] Planck Collaboration, P. A. R. Ade et al., "Planck 2015 results. XIII. Cosmological parameters", arXiv:1502.01589.

[7] A. H. Guth, "The Inflationary Universe: A Possible Solution to the Horizon and Flatness Problems", Phys.Rev. D23 (1981) 347-356.

[8] A. Albrecht and P. J. Steinhardt, "Cosmology for Grand Unified Theories with Radiatively Induced Symmetry Breaking", Phys.Rev.Lett. 48 (1982) 1220-1223.

[9] A. D. Linde, "A New Inflationary Universe Scenario: A Possible Solution of the Horizon, Flatness, Homogeneity, Isotropy and Primordial Monopole Problems", Phys.Lett. B108 (1982) 389-393.

[10] A. D. Linde, "Chaotic Inflation", Phys.Lett. B129 (1983) 177-181.

[11] A. R. Liddle and D. Lyth, "Cosmological inflation and large scale structure", Cambridge University Press, 2000.

[12] M. J. White and D. Scott, "Why not consider closed universes?", Astrophys. J. 459 (1996) 415, arXiv:astro-ph/9508157.

[13] S. Gratton, A. Lewis, and N. Turok, "Closed universes from cosmological instantons", Phys. Rev. D65 (2002) 043513, arXiv:astro-ph/0111012. 
[14] A. Lewis, A. Challinor, and A. Lasenby, "Efficient computation of CMB anisotropies in closed FRW models", Astrophys. J. 538 (2000) 473-476, astro-ph/9911177.

[15] Planck Collaboration, P. A. R. Ade et al., "Planck 2013 results. XVI. Cosmological parameters", Astron. Astrophys. 571 (2014) A16, arXiv:1303.5076.

[16] D. J. Schwarz, C. J. Copi, D. Huterer, and G. D. Starkman, "CMB Anomalies after Planck", 2015. arXiv:1510.07929.

[17] P. Hunt and S. Sarkar, "Search for features in the spectrum of primordial perturbations using Planck and other datasets", JCAP 1512 (2015), no. 12, 052, arXiv:1510.03338.

[18] A. D. Linde, "Can we have inflation with $\Omega>1$ ?", JCAP 0305 (2003) 002, arXiv:astro-ph/0303245.

[19] J. White, Y.-l. Zhang, and M. Sasaki, "Scalar suppression on large scales in open inflation", Phys. Rev. D90 (2014), no. 8, 083517, arXiv:1407.5816.

[20] C. R. Contaldi, M. Peloso, L. Kofman, and A. D. Linde, "Suppressing the lower multipoles in the CMB anisotropies", JCAP 0307 (2003) 002, arXiv:astro-ph/0303636.

[21] R. K. Jain, P. Chingangbam, J.-O. Gong, L. Sriramkumar, and T. Souradeep, "Punctuated inflation and the low CMB multipoles", JCAP 0901 (2009) 009, arXiv:0809.3915.

[22] F. G. Pedro and A. Westphal, "Low- $\ell$ CMB power loss in string inflation", JHEP 04 (2014) 034, arXiv:1309.3413.

[23] L. Lello and D. Boyanovsky, "Tensor to scalar ratio and large scale power suppression from pre-slow roll initial conditions", JCAP 1405 (2014) 029, arXiv:1312.4251.

[24] L. Lello, D. Boyanovsky, and R. Holman, "Pre-slow roll initial conditions: large scale power suppression and infrared aspects during inflation", Phys. Rev. D89 (2014), no. 6, 063533, arXiv:1307.4066.

[25] J. L. Cook and L. M. Krauss, "Large Slow Roll Parameters in Single Field Inflation", arXiv:1508.03647.

[26] J.-P. Uzan, U. Kirchner, and G. F. R. Ellis, "WMAP data and the curvature of space", Mon. Not. Roy. Astron. Soc. 344 (2003) L65, arXiv:astro-ph/0302597.

[27] G. F. R. Ellis, S. J. Stoeger, William R., P. McEwan, and P. Dunsby, "Dynamics of inflationary universes with positive spatial curvature", Gen. Rel. Grav. 34 (2002) 1445-1459, arXiv:gr-qc/0109023.

[28] G. F. R. Ellis, P. McEwan, S. J. Stoeger, William R., and P. Dunsby, "Causality in inflationary universes with positive spatial curvature", Gen. Rel. Grav. 34 (2002) 1461-1481, arXiv:gr-qc/0109024.

[29] G. Efstathiou, "Is the low CMB quadrupole a signature of spatial curvature?", Mon. Not. Roy. Astron. Soc. 343 (2003) L95, arXiv:astro-ph/0303127.

[30] A. Lasenby and C. Doran, "Closed universes, de Sitter space and inflation", Phys. Rev. D71 (2005) 063502, arXiv:astro-ph/0307311.

[31] J. P. Luminet, J. Weeks, A. Riazuelo, R. Lehoucq, and J. P. Uzan, "Dodecahedral space topology as an explanation for weak wide - angle temperature correlations in the cosmic microwave background", Nature 425 (2003) 593, arXiv:astro-ph/0310253.

[32] E. Masso, S. Mohanty, A. Nautiyal, and G. Zsembinszki, "Imprint of spatial curvature on inflation power spectrum", Phys. Rev. D78 (2008) 043534, arXiv:astro-ph/0609349.

[33] D. Langlois, "Hamiltonian formalism and gauge invariance for linear perturbations in inflation", Class. Quant. Grav. 11 (1994) 389-407. 
[34] J. D. Barrow, "The Premature Recollapse Problem in Closed Inflationary Universes", Nucl. Phys. B296 (1988) 697-709.

[35] J. J. Halliwell and S. W. Hawking, "The Origin of Structure in the Universe", Phys. Rev. D31 (1985) 1777.

[36] M. Fernandez-Mendez, G. A. M. Marugan, J. Olmedo, and J. M. Velhinho, "Unique Fock quantization of scalar cosmological perturbations", Phys. Rev. D85 (2012) 103525, arXiv: 1203.2525

[37] J. D. Barrow and S. Cotsakis, "Inflation and the Conformal Structure of Higher Order Gravity Theories", Phys. Lett. B214 (1988) 515-518.

[38] K.-i. Maeda, "Towards the Einstein-Hilbert Action via Conformal Transformation", Phys. Rev. D39 (1989) 3159.

[39] A. A. Starobinsky, S. Tsujikawa, and J. Yokoyama, "Cosmological perturbations from multifield inflation in generalized Einstein theories", Nucl. Phys. B610 (2001) 383-410, arXiv:astro-ph/0107555.

[40] A. De Felice and S. Tsujikawa, "f(R) theories", Living Rev. Rel. 13 (2010) 3, arXiv:1002.4928.

[41] J. M. Bardeen, "Gauge Invariant Cosmological Perturbations", Phys. Rev. D22 (1980) 1882-1905.

[42] V. F. Mukhanov, H. A. Feldman, and R. H. Brandenberger, "Theory of cosmological perturbations. Part 1. Classical perturbations. Part 2. Quantum theory of perturbations. Part 3. Extensions", Phys. Rept. 215 (1992) 203-333.

[43] J. Goldberg, E. T. Newman, and C. Rovelli, "On Hamiltonian systems with first-class constraints", Journal of Mathematical Physics 32 (1991) 2739-2743.

[44] V. Mukhanov and S. Winitzki, "Introduction to quantum effects in gravity", Cambridge University Press, 2007.

[45] S. A. Fulling, "Aspects of Quantum Field Theory in Curved Space-time", London Math. Soc. Student Texts 17 (1989) 1-315.

[46] B. Bonga and B. Gupt, "Phenomenological investigation of a quantum gravity extension of inflation with the Starobinsky potential", Phys. Rev. D93 (2016), no. 6, 063513, arXiv:1510.04896.

[47] B. Bonga, B. Gupt, and N. Yokomizo, "Quantum gravity extension of inflationary scenario in closed FLRW model and the CMB", in preparation.

[48] A. Lewis and S. Bridle, "Cosmological parameters from CMB and other data: A Monte Carlo approach", Phys. Rev. D66 (2002) 103511, arXiv:astro-ph/0205436.

[49] A. Ashtekar and D. Sloan, "Loop quantum cosmology and slow roll inflation", Phys. Lett. B694 (2011) 108-112, arXiv:0912.4093.

[50] A. Ashtekar and D. Sloan, "Probability of Inflation in Loop Quantum Cosmology", Gen. Rel. Grav. 43 (2011) 3619-3655, arXiv:1103.2475.

[51] A. Corichi and A. Karami, "On the measure problem in slow roll inflation and loop quantum cosmology", Phys. Rev. D83 (2011) 104006, arXiv:1011.4249.

[52] A. Ashtekar, T. Pawlowski, P. Singh, and K. Vandersloot, "Loop quantum cosmology of k=1 FRW models", Phys. Rev. D75 (2007) 024035, arXiv:gr-qc/0612104.

[53] A. Ashtekar, W. Kaminski, and J. Lewandowski, "Quantum field theory on a cosmological, quantum space-time", Phys. Rev. D79 (2009) 064030, arXiv:0901.0933.

[54] I. Agullo, A. Ashtekar, and W. Nelson, "Extension of the quantum theory of cosmological perturbations to the Planck era", Phys. Rev. D87 (2013), no. 4, 043507, arXiv:1211.1354.

[55] B. Bonga, B. Gupt, and N. Yokomizo, "Inflation in closed FLRW model: Tensor perturbations", in preparation. 
[56] F. Schmidt and L. Hui, "Cosmic Microwave Background Power Asymmetry from Non-Gaussian Modulation", Phys. Rev. Lett. 110 (2013) 011301, arXiv:1210.2965, [Erratum: Phys. Rev.

Lett.110,059902(2013)].

[57] I. Agullo, "Loop quantum cosmology, non-Gaussianity, and CMB power asymmetry", Phys. Rev. D92 (2015) 064038, arXiv:1507.04703.

[58] S. Adhikari, S. Shandera, and A. L. Erickcek, "Large-scale anomalies in the cosmic microwave background as signatures of non-Gaussianity", Phys. Rev. D93 (2016), no. 2, 023524, arXiv:1508.06489.

[59] L. F. Abbott and R. K. Schaefer, "A general, gauge-invariant analysis of the cosmic microwave anisotropy", The Astrophysical Journal 308 (1986) 546-562. 